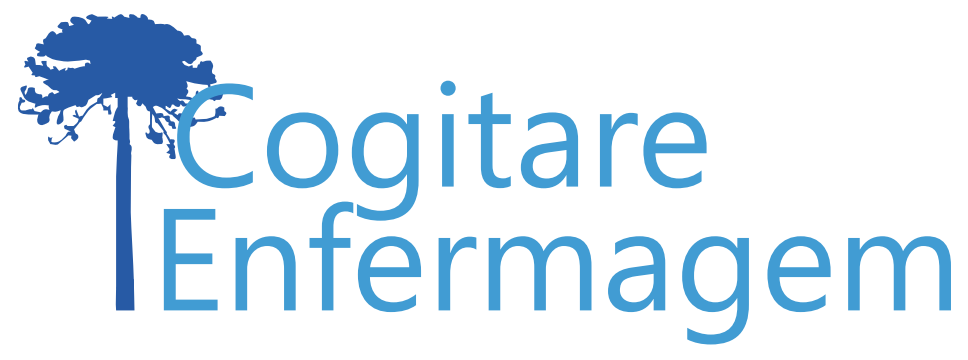

\title{
COBERTURA DE TRATAMENTO DIRETAMENTE OBSERVADO SEGUNDO O RISCO DE COINFECÇÃO TB/HIV E DESFECHOS DESFAVORÁVEIS*
}

Laura Terenciani Campoy', Luiz Henrique Arroyo², Antônio Carlos Vieira Ramos ${ }^{3}$, Rubia Laine de Paula Andrade ${ }^{4}$, Marcos Augusto Moraes Arcoverde ${ }^{5}$, Josilene Dália Alves ${ }^{6}$, Ricardo Alexandre Arcêncio ${ }^{7}$

\section{RESUMO}

Objetivo: analisar a cobertura do tratamento diretamente observado segundo o risco de coinfecção tuberculose/vírus da imunodeficiência humana e desfechos desfavoráveis.

Método: estudo ecológico com dados secundários relacionados aos 10.389 casos novos de coinfecção notificados no estado de São Paulo de 2010 a 2015. Dados analisados pelo Índice Local de Moran, estatística de varredura espacial e Modelos Bayesianos Hierárquicos.

Resultados: região metropolitana de São Paulo e Baixada Santista concentraram maior incidência de coinfecção e abandono ao tratamento. Baixa cobertura de tratamento diretamente observado esteve associada aos territórios em risco para a coinfecção e maior risco de abandono. Município de São Paulo, região litorânea e região de Ribeirão Preto apresentaram maior incidência de óbito, o qual não apresentou relação com a cobertura do tratamento diretamente observado.

Conclusão: baixa cobertura de tratamento diretamente observado apresentou associação com maior risco de coinfecção e abandono do tratamento.

DESCRITORES: Tuberculose; Síndrome de Imunodeficiência Adquirida; Coinfecção; Pesquisa sobre Serviços de Saúde; Pesquisa Operacional.

*Artigo extraído da tese de doutorado "Análise geoepidemiológica e operacional da coinfecção TB/HIV". Universidade de São Paulo, 2019.

\section{COMO REFERENCIAR ESTE ARTIGO:}

Campoy LT, Arroyo LH, Ramos ACV, Andrade RL de P, Arcoverde MAM, Alves JD, et al. Cobertura de tratamento diretamente observado segundo o risco de coinfecção TB/HIV e desfechos desfavoráveis. Cogitare enferm. [Internet]. 2019 [acesso em "colocar data de acesso, dia, mês abreviado e ano"]; 24. Disponível em: http:// dx.doi.org/10.5380/ce.v24i0.66775.

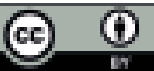

Este obra está licenciado com uma Licença Creative Commons Atribuição 4.0 Internacional.

${ }^{1}$ Enfermeira. Doutoranda em Enfermagem em Saúde Pública. Universidade de São Paulo. Ribeirão Preto, SP, Brasil. $\odot$ ${ }^{2}$ Fisioterapeuta. Doutorando em Enfermagem. Universidade de São Paulo. Ribeirão Preto, SP, Brasil. $(0)$

${ }^{3}$ Enfermeiro. Doutorando em Enfermagem em Saúde Pública. Universidade de São Paulo. Ribeirão Preto, SP, Brasil. ${ }^{4}$ Enfermeira. Doutora em Enfermagem. Especialista de laboratório da Escola de Enfermagem de Ribeirão Preto Universidade de São Paulo. Ribeirão Preto, SP, Brasil. $($ )

${ }^{5}$ Enfermeiro. Doutor em Enfermagem. Docente da Universidade Estatual do Oeste do Paraná. Foz do Iguaçu, PR, Brasil. 9 ${ }^{6}$ Enfermeira. Doutora em Enfermagem. Docente do Instituto Federal do Mato Grosso. Barra do Garça, MT, Brasil. ()

${ }^{7}$ Enfermeiro. Doutor em Enfermagem. Docente de Enfermagem da Universidade de São Paulo. Ribeirão Preto, SP, Brasil. $(9$ 


\title{
COVERAGE OF DIRECTLY OBSERVED TREATMENT ACCORDING TO THE RISK OF TUBERCULOSIS/HIV COINFECTION AND UNFAVORABLE OUTCOMES
}

\begin{abstract}
Objective: To analyze the coverage of directly observed treatment according to the risk of tuberculosis/human immunodeficiency virus coinfection and unfavorable outcomes.

Methods: Ecological study with secondary data related to 10,389 new cases of coinfection notified in the state of São Paulo, Brazil, from 2010 to 2015. They were analyzed by applying local Moran's index, spatial scan statistics, and hierarchical Bayesian models.

Results: The São Paulo metropolitan area and Baixada Santista concentrate the highest incidence of coinfection and treatment default. Low coverage of directly observed treatment was associated with areas at risk for the coinfection and higher withdrawal risk. The city of São Paulo, the coastal region, and the Ribeirão Preto area showed a higher incidence of deaths, which did not show an association with the coverage of directly observed treatment. Conclusion: Low coverage of directly observed treatment was associated with a higher risk of coinfection and treatment default.
\end{abstract}

DESCRIPTORS: Tuberculosis; Acquired Immunodeficiency Syndrome; Coinfection; Health Services Research; Operations Research.

\section{COBERTURA DE TRATAMIENTO DIRECTAMENTE OBSERVADO SEGUN RIESGO DE COINFECCION TB/VIH Y DESENLACES DESFAVORABLES}

\begin{abstract}
RESUMEN
Objetivo: Analizar la cobertura del tratamiento directamente observado según el riesgo de coinfección tuberculosis/virus de la inmunodeficiencia humana y desenlaces desfavorables. Método: Estudio ecológico con datos secundarios relacionados con los 10.389 nuevos casos de coinfección informados en el estado de São Paulo de 2010 a 2015. Datos analizados mediante Índice Local de Moran, estadística de muestreo espacial y Modelos Bayesianos Jerárquicos.

Resultados: La región metropolitana de São Paulo y Baixada Santista concentraron mayor incidencia de coinfección y abandono del tratamiento. La baja cobertura de tratamiento directamente observado estuvo asociada a las áreas con riesgo de coinfección y mayor grado de abandono. El Municipio de São Paulo, la región litoral y el área de Ribeirão Preto expresaron mayor incidencia de decesos, no relacionándose ello con la cobertura del tratamiento directamente observado.

Conclusión: La baja cobertura de tratamiento directamente observado mostró asociación con mayor riesgo de coinfección y abandono del tratamiento.
\end{abstract}

DESCRIPTORES: Tuberculosis; Síndrome de Inmunodeficiencia Adquirida; Coinfección; Investigación sobre Servicios de Salud; Investigación Operativa. 
A tuberculose (TB) permanece como grave problema de saúde pública, afligindo especificamente os países em desenvolvimento, tornando sua eliminação cada vez mais distante da meta de menos de 1 caso por 100.000 habitantes, estabelecida pela estratégia End $T B^{(1)}$.

Mundialmente, em 2015 foram notificados 10,4 milhões de casos de TB, sendo que 1,1 milhão (10,6\%) desses eram pessoas vivendo com vírus da imunodeficiência humana - HIV/AIDS (PVHA) $)^{(1)}$. Nesse panorama, o Brasil ocupa a $20^{a}$ posição quanto à carga da doença e a $19^{\mathrm{a}}$ no que se refere à coinfecção TB/HIV(2).

A coinfecção TB/HIV constitui uma das principais barreiras para o controle da TB ${ }^{(3)}$, uma vez que a presença de ambas condições acarreta maior chance de desfechos desfavoráveis, como abandono do tratamento ou óbito ${ }^{(4)}$. Em 2015, entre pacientes coinfectados TB/HIV, a taxa de abandono foi de $11,6 \%$ e a taxa de óbito foi de $17,8 \%$, valores muito distantes das metas preconizadas pela OMS de $5 \%$ de abandono e $1 \%$ de óbito ${ }^{(5)}$.

Contudo, a estratégia de Tratamento Diretamente Observado (TDO) pode ser uma aliada nesta direção, constituindo um recurso para se promover a adesão e o sucesso do tratamento(6), uma vez que garante a ingestão medicamentosa pelo paciente e o fortalecimento do vínculo deste com o serviço e profissional de saúde ${ }^{(7)}$. No país, a taxa de cura está em torno de 70,4\%, no entanto, a implantação do TDO possibilitaria ampliar as chances de cura para $90 \%$ dos casos e alcançar a meta estabelecida de $85 \%{ }^{(4)}$.

Considerando os desafios para a eliminação da TB, torna-se importante o desenvolvimento de estudos que possam evidenciar a cobertura do TDO e seu impacto no desfecho de tratamento. Assim, este estudo teve como objetivo analisar a cobertura do TDO em municípios do estado de São Paulo (ESP) segundo o risco de coinfecção TB/HIV e desfechos desfavoráveis.

\section{MÉTODO}

Trata-se de um estudo ecológico ${ }^{(8)}$, realizado nos 645 municípios do ESP, o qual apresenta cerca de 9,9\% (1.963) dos casos com coinfecção TB/HIV do país ${ }^{(9)}$.

A população do estudo atende aos seguintes critérios de inclusão: casos novos notificados de ŢB com coinfecção por HIV e residentes no ESP entre 2010 a 2015. Definiuse como critérios de exclusão: indivíduos do sistema prisional, casos que tiveram como desfecho falência, mudança de diagnóstico e transferência para outros estados. Os dados foram colhidos em dezembro de 2017 no Sistema de Notificação e Acompanhamento dos Casos de TB (TBWEB) e nas bases públicas do Instituto Brasileiro de Geografia e Estatística. $O$ tratamento dos dados obtidos seguiu a exclusão de casos conforme critérios de exclusão e daqueles com exame anti-HIV negativo, sem informação e em andamento.

Para a análise dos dados, calculou-se a incidência anual dos casos de coinfecção TB/ HIV realizando a padronização direta pelas covariáveis sexo e idade ( $\leq 14$ anos, 15 a 59 anos e $\geq 60$ anos) dos pacientes, e considerando como população padrão a população total do estado ${ }^{(10)}$.

As proporções de desfechos desfavoráveis (abandono ou óbito) dos casos de coinfecção TB/HIV foram calculadas por município. Em relação ao óbito, foram incluídos óbitos por TB e não TB, uma vez que estes últimos são em sua grande maioria óbitos por HIV.

Desse modo, foi atribuído a cada município uma taxa de incidência e a proporção de desfechos desfavoráveis, sendo posteriormente produzido mapas coropléticos no ArcGis 
10.6. Na construção dos intervalos de incidência nos referidos mapas, definiu-se o primeiro intervalo como sendo a ausência de casos registrados no município, e os outros, seguindo o método de aproximação denominado "Quebra Natural" (Natural Breaks) desenvolvido por Jenks ${ }^{(11)}$.

Para identificar os territórios de risco para a incidência dos casos e desfechos desfavoráveis, recorreu-se inicialmente ao Índice Local de Moran (LISA). O LISA consiste em uma análise de localização de aglomerados espaciais que considera tanto o efeito dos municípios vizinhos, como os valores médios de toda a área de estudo, ou seja, do ESP(12).

Os resultados classificam os territórios como áreas quentes (Alto-Alto), áreas frias (Baixo-Baixo) e outliers espaciais (Alto-Baixo e Baixo-Alto). Apenas as áreas Alto-Alto e Baixo-Baixo foram consideradas para a elaboração de mapas e análises subsequentes.

Essas análises requerem um parâmetro de relação espacial (peso espacial), ou seja, um modelo de interação entre as localidades estudadas taxa de incidência e a proporção de desfechos desfavoráveis. A interpolação espacial é fundamental para compreender os padrões espaciais ${ }^{(13)}$ e pode influenciar consideravelmente os resultados encontrados ${ }^{(14)}$, de tal forma que foram utilizados três modelos distintos para ampliar a capacidade de detecção de aglomerados.

O primeiro, denominado Ponderação do Inverso das Distâncias (Inverse Distance Weighting), utiliza as distâncias entre as unidades analisadas como elemento de ponderação do peso espacial. Assim, todos os municípios se influenciam, mas os municípios mais próximos possuem mais peso nessa relação(15).

O segundo e terceiro método determinam o grau de relação espacial baseado nas fronteiras das unidades de análise. Esse método é conhecido como Vizinhos Naturais ou Contiguidade Espacial e foi desenvolvida por Sibson $(1981)^{(16)}$. O primeiro tipo deste modelo é denominado Rook e o segundo, Queen.

Após a abordagem anterior, aplicou-se a estatística de varredura espacial(17), que realiza a contagem de casos de coinfecção TB/HIV ou dos que abandonaram o tratamento ou foram a óbito por cada unidade de análise. Deste modo, a identificação dos territórios de risco por este tipo de análise é realizada considerando uma distribuição de Poisson, sendo ainda exercida uma padronização pelas covariáveis sexo e idade.

A varredura possibilitou a classificação dos territórios de risco a partir do Risco Relativo (RR) e seus respectivos intervalos de confiança de 95\% (IC 95\%). Utilizou-se o software SaTScan versão 9.4, com a ausência de sobreposição dos territórios de risco, 999 replicações para simulação de Monte Carlo e territórios de risco com formatos circulares.

Na definição do tamanho máximo dos aglomerados, utilizou-se a proposta de Han et al. (2016)(18), que permitiu determinar como população máxima na janela circular de análise $30 \%$ da população em risco nos três eventos analisados.

Em seguida, para realização dos modelos bayesianos hierárquicos, os 645 municípios foram categorizados como pertencentes ou não a um dos territórios de risco para a incidência, abandono de tratamento e óbito. Especificamente no caso do LISA, foram considerados territórios de risco aqueles pertencentes à classificação Alto-Alto. Dessa forma, definindo esta como variável resposta, recorreu-se à regressão logística com distribuição Binomial, ao qual se verificou a relação entre os territórios de risco e a cobertura do TDO por município; estes foram classificados em três categorias: alta, $\geq 80 \%$ de cobertura do TDO; mediana, $31 \%$ a $79 \%$; e baixa, $\leq 30 \%$; foram utilizados os dados do TDO indicado e não efetivado.

Ainda neste modelo, foi considerada a ausência de preenchimento desta informação no TBWEB ("missing data") por município, com objetivo de retirar possíveis efeitos de confusão na análise. 
Após a regressão logística, foi considerada também a inserção do efeito espacial a partir do modelo Besag, York, Mollié (19), o qual considera efeitos aleatórios específicos da área por meio de dois componentes: efeito que varia de forma estruturada no espaço, ou seja, aglomeração ou heterogeneidade correlacionada; efeitos que variam de forma não estruturada entre as áreas de análise (heterogeneidade não correlacionada). Os modelos com e sem a componente espacial foram comparados a partir dos valores de WatanabeAkaike information criterion (WAIC), sendo selecionado aquele modelo com menor valor do estimador.

Foram realizados modelos estatísticos para cada variável resposta, incidência de casos de coinfecção TB/HIV e dos casos que abandonaram o tratamento ou foram a óbito, além do cálculo do Odds Ratio (OR) e Intervalo de Credibilidade de 95\% (IC 95\%) para cada variável explicativa. Para as análises foram consideradas prioris não informativas e as distribuições posteriores foram obtidas utilizando o Integrated Nested Laplace Approximation (INLA), disponível no software R pelo pacote R-INLA ${ }^{(20)}$.

A pesquisa foi aprovada pelo Comitê de Ética em Pesquisa da Escola de Enfermagem de Ribeirão Preto da Universidade de São Paulo, número 1.617.513.

\section{RESULTADOS}

Foram levantados 10.389 casos de coinfeccão TB/HIV, sendo 7.479 (72\%) do sexo masculino e 2.910 (28\%) feminino, $9.980(96,1 \%)$ na faixa etária de 14 a 59 anos. Dos municípios que apresentaram casos, estes estavam localizados na Capital (SPC) e região metropolitana de São Paulo (RMSP) (6.290 - 60,5\%), interior (3.113 - 30,0\%) e litoral (986 $9,5 \%)$. Em relação ao desfecho, $5.847(56,3 \%)$ casos obtiveram cura, $2.396(23,1 \%)$ foram a óbito e $2.047(19,7 \%)$ abandonaram o tratamento.

A distribuição espacial das taxas de incidência dos casos de coinfecção TB/HIV e a proporção de desfechos desfavoráveis do tratamento da TB podem ser observadas na Figura 1. 


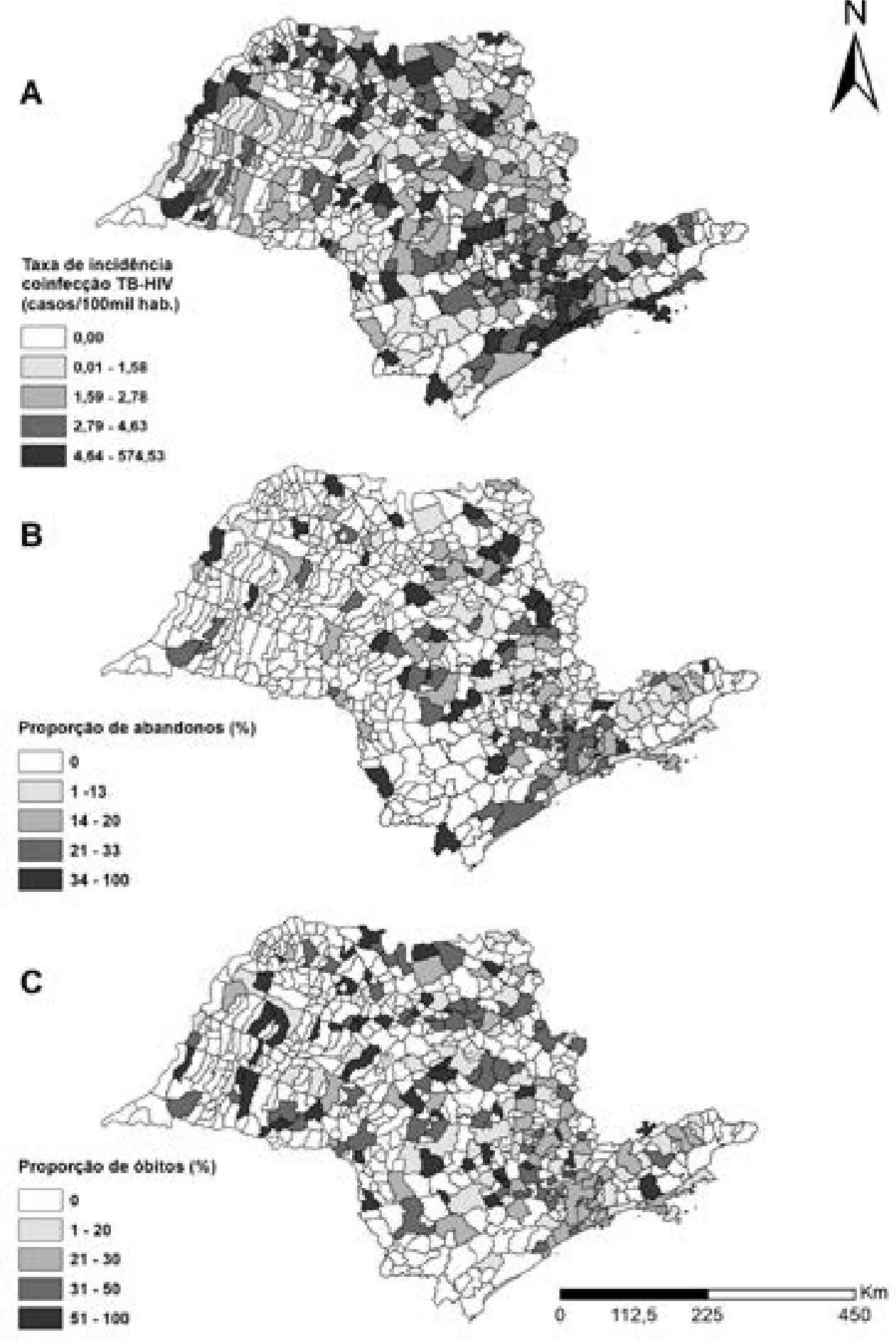

Figura 1 - Padrão espacial da coinfecção TB/HIV e desfechos desfavoráveis no estado de São Paulo (20102015). Ribeirão Preto, SP, Brasil, 2019

Legenda: A - Incidência de coinfecção TB/HIV (casos/100 mil habitantes). B - Proporção de casos de coinfecção TB/HIV que abandonaram o tratamento da tuberculose (\%). C - Proporção de casos de coinfecção TB/HIV que foram a óbito (\%).

As taxas de incidência mais elevadas se concentram na RMSP e Baixada Santista, todavia observa-se que municípios do interior apresentam taxas igualmente elevadas sem necessariamente serem cercados com municípios semelhantes. Destaca-se que 13 (2\%) municípios do estado apresentam taxas de incidência maiores de 20 por 100.000 habitantes. 
Em relação aos desfechos desfavoráveis para coinfecçãoTB/HIV, observa-se um padrão similar de distribuição dos abandonos em comparação à incidência dos casos, ou seja, os municípios mais próximos da RMSP apresentam uma maior frequência de abandonos. Em contrapartida, a distribuição dos casos que foram a óbito no estado aparentemente possui um padrão aleatório, isso devido a municípios do interior apresentar elevadas proporções de indivíduos que evoluíram para óbito sem possuírem vizinhos semelhantes. Entretanto, é importante salientar que a RMSP apresentou certa concentração do evento.

No Índice de Moran Local, no que se refere à incidência dos casos (Figura 2), a RMSP apresentou-se um aglomerado alto-alto para os três métodos de interpolação espacial. $O$ aglomerado baixo-baixo mostrou-se com maior número de municípios e localizou-se com maior evidência na região noroeste do estado.
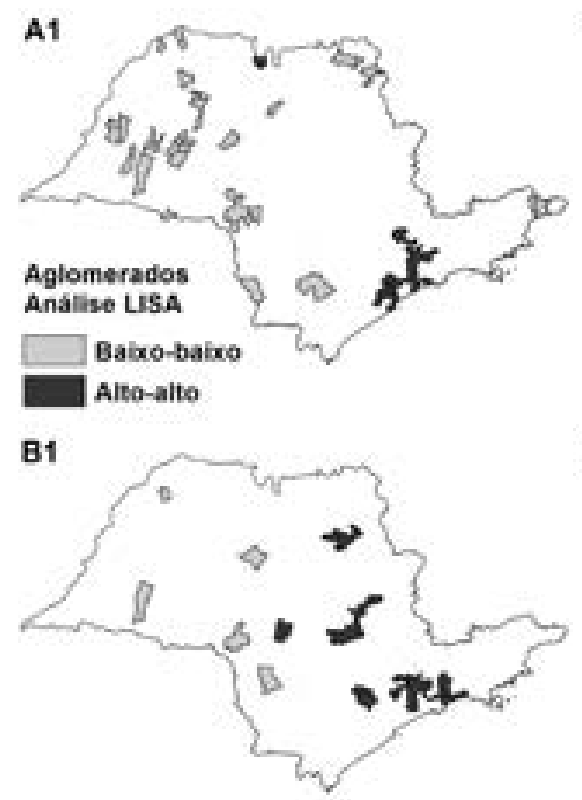

C1

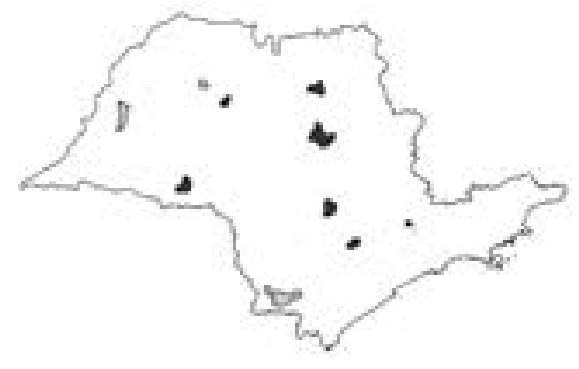

A2

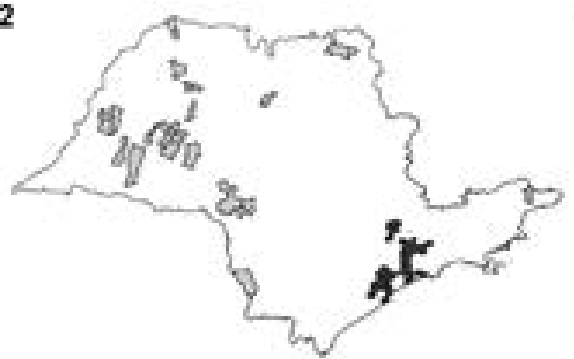

B2

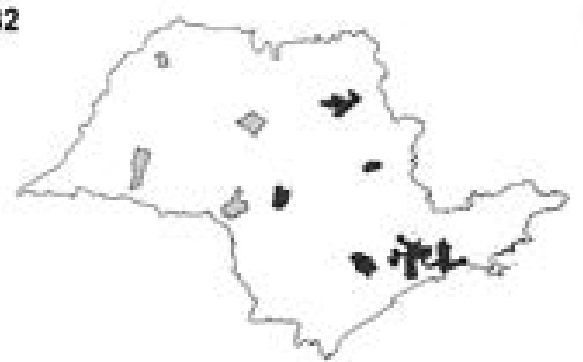

C2

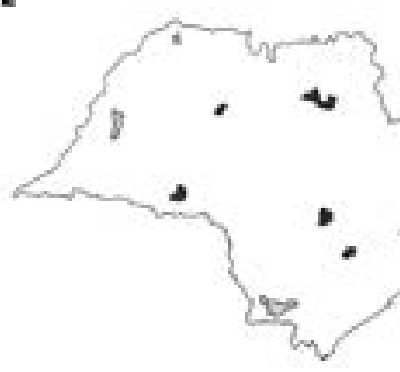

A3

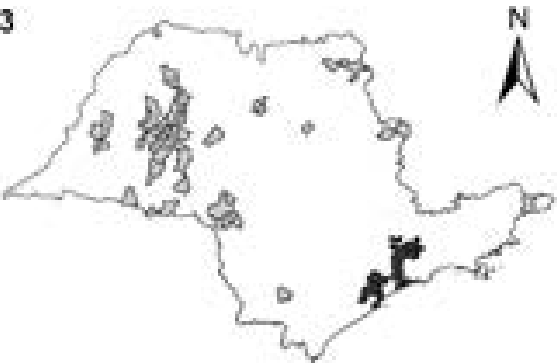

B3

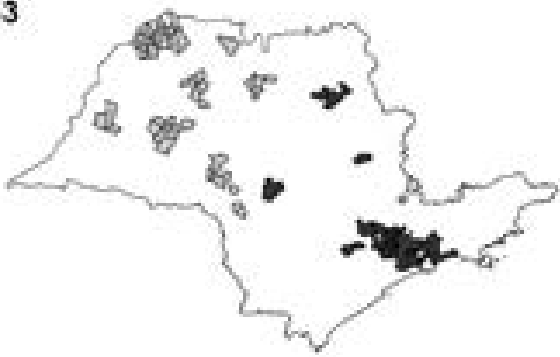

C3

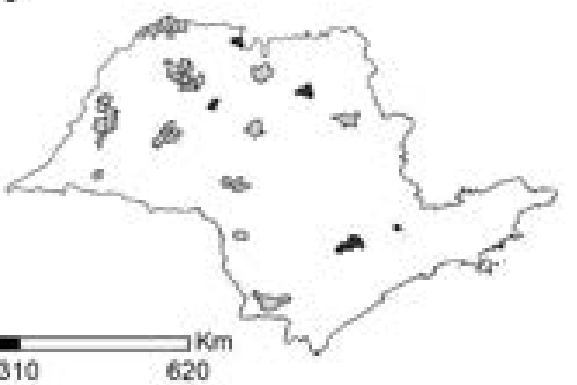

Figura 2 - Distribuição espacial das incidências de coinfecção TB/HIV e proporções dos desfechos desfavoráveis no estado de São Paulo (2010-2015). Ribeirão Preto, SP, Brasil, 2019

Legenda: A - Taxa de incidência de coinfecção TB/HIV (casos/100 mil habitantes). B - Proporção de casos de coinfecção TB/HIV que abandonaram o tratamento da tuberculose (\%). C - Proporção de casos de coinfecção TB/HIV que foram a óbito (\%). A1, B1, C1 - Contiguity edges cornes; A2, B2, C2 - Contiguity edges only; A3, B3, C3 - Inverse distance.

A análise LISA para o abandono apresentou aglomerado alto-alto na RMSP, região de Ribeirão Preto e municípios de Lençóis Paulista e Leme. Os municípios de Rio Claro e Piracicaba foram considerados como parte do aglomerado alto-alto. O aglomerado baixobaixo foi mais evidenciado na região noroeste e divisa com o estado de Minas Gerais. 
$\mathrm{Na}$ análise para a ocorrência de óbito nos casos de coinfecção TB/HIV, foram identificados alguns municípios significativos para o aglomerado alto-alto. Para o aglomerado baixo-baixo, foi evidenciada a região noroeste.

$\mathrm{Na}$ análise de varredura espacial dos casos, quatro territórios de risco foram identificados (Figura 3); o território de risco 1 está localizado em SPC, enquanto que o segundo envolveu a região litorânea do estado. No interior foram evidenciados dois territórios de risco, sendo um englobando Ribeirão Preto, Cravinhos e Dummont (território de risco 3) e São José do Rio Preto (território de risco 4).
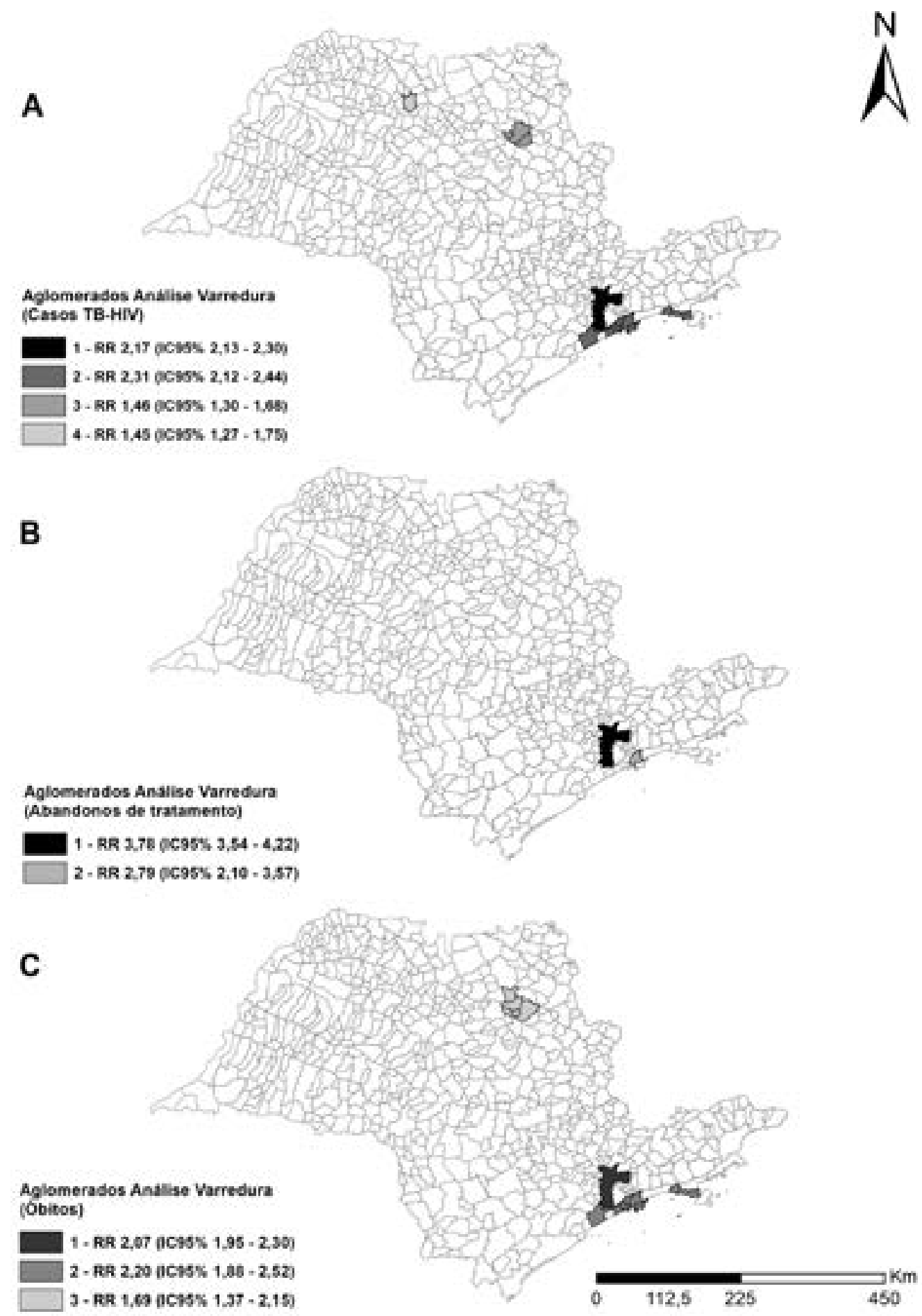

Figura 3 - Áreas críticas para a coinfecção TB/HIV e para abandono do tratamento da tuberculose e óbito entre casos de coinfecção no estado de São Paulo (2010-2015). Ribeirão Preto, SP, Brasil, 2019 
Legenda: A - Territórios de risco para a incidência de coinfecção TB/HIV. B - Territórios de risco para abandono do tratamento da tuberculose em casos de coinfecção TB/HIV (\%). C - Territórios de risco para óbito em casos de coinfecção TB/HIV (\%).

Em termos de abandono do tratamento, a varredura identificou dois territórios de risco: SPC e Santos. Em relação aos óbitos foram evidenciadas três áreas de risco: SPC, região litorânea do estado e região de Ribeirão Preto.

Seguidamente, através das análises Bayesianas (Tabela 1), verificaram-se os três modelos propostos tendo como variáveis respostas os territórios de risco para incidência, abandono de tratamento e óbito, sendo que para todas as análises o modelo elaborado com a componente espacial foi aquele com melhor estimador WAIC.

Tabela 1 - Resultados da regressão associando territórios de risco para a incidência e desfechos desfavoráveis dos casos TB/HIV e Tratamento Diretamente Observado. Ribeirão Preto, SP, Brasil, 2019

Variável explicativa

Odds Ratio (Intervalo de Credibilidade de 95\%)

\begin{tabular}{ccc}
\hline $\begin{array}{c}\text { Territórios } \\
\text { de risco para } \\
\text { incidência* }\end{array}$ & $\begin{array}{c}\text { Territórios } \\
\text { de risco para } \\
\text { abandono }\end{array}$ & $\begin{array}{c}\text { Territórios de } \\
\text { risco para óbito* }\end{array}$ \\
\hline
\end{tabular}

Cobertura do Tratamento Diretamente

Observado

\begin{tabular}{lccc}
\hline$\geq 80 \%$ & 1 & 1 & 1 \\
\hline $31 \%-79 \%$ & $1,10(0,31-4,10)$ & $3,39(1,03-12,15)$ & $2,92(0,94-9,04)$ \\
\hline$\leq 30 \%$ & $0,18(0,03-0,76)$ & $0,13(0,02-0,57)$ & $0,01(0,01-9,30)$ \\
\hline Ausência de preenchimento na ficha de & $3,81(0,30-35,61)$ & $1,33(0,09-14,62)$ & $0,78(0,02-18,49)$
\end{tabular}

notificação acerca do tipo de tratamento

(variável contínua)

*Watanabe-Akaike Information Criterion (WAIC) com componente espacial=133,73; sem componente espacial = 187,98. Estrutura especial explicou $36 \%$ da variabilidade dos dados.

tWAIC com componente espacial= 145,89; sem componente espacial $=250,93$. Estrutura especial explicou $30 \%$ da variabilidade dos dados.

tWAIC com componente espacial= 253,14; sem componente espacial $=263,71$. Estrutura especial explicou $97 \%$ da variabilidade dos dados.

No que concerne à incidência, tendo como valor de referência cobertura de TDO igual ou acima de $80 \%$ dos casos de coinfecção, observou-se uma relação entre os territórios de risco e a baixa cobertura do TDO (OR=0,18; IC95\%=0,03-0,76). No caso do abandono, evidenciou-se associação entre território de risco deste desfecho com cobertura mediana do TDO $(\mathrm{OR}=3,39 ; \mathrm{IC} 95 \%=1,03-12,15)$ e valores de baixa cobertura $(\mathrm{OR}=0,13$; IC95\% $=0,02-0,57)$, tendo novamente como valor de referência a cobertura igual ou acima de $80 \%$. Por fim, para os territórios de risco de óbito, não foi identificada evidências de relação com a cobertura do TDO. 
As áreas que apresentaram territórios de risco nas análises de agrupamento se destacam por serem áreas populosas, com grandes desigualdades socioeconômicas e com intensa movimentação urbana (21). Habitualmente, grandes centros urbanos apresentam maior número de notificações de casos de TB, fato coincidente com a maior ocorrência de fatores de risco para a ocorrência e para desfechos desfavoráveis da TB, como abuso de drogas injetáveis, migração e falta de moradia e desigualdade social.

Somado a isto, estas são consideradas regiões turísticas e portuárias, tanto por se tratar da capital do estado e maior polo econômico do país, como por serem regiões litorâneas, o que pode dificultar as ações de controle da coinfecção. Esses resultados coincidem com estudo realizado em Portugal(22), em que se identificou que as principais áreas urbanas que pertenciam a região turística apresentam as maiores taxas para o abandono do tratamento dos casos de TB.

As diferentes análises espaciais realizadas desempenharam um papel de maior abrangência no rastreio de territórios de risco para a incidência de coinfecção TB/HIV e desfechos desfavoráveis. Tal abordagem amplia a compreensão da distribuição da coinfecção no estado e apresenta possíveis regiões críticas que necessitam de ações interventivas. Para a incidência e o abandono, foi observada a formação dos maiores territórios de risco no ESP, o que pode representar que características inerentes a estas regiões são de grande importância para a distribuição espacial dos casos e abandonos de tratamento.

$\mathrm{Na}$ análise de associação dos territórios de risco com a cobertura do TODO, foi identificada uma importante relação. Sabe-se que o TDO é mais efetivo do que a modalidade autoadministrada, para o sucesso do tratamento(23). Apesar dos desafios encontrados para sua operacionalização, como a organização dos serviços de saúde, do contexto geopolítico e gerencial, e da necessidade de adaptação dos profissionais de saúde e governos locais, observam-se melhorias nos indicadores de controle da TB a partir dessa política, particularmente entre populações vulneráveis ${ }^{(24)}$.

O Ministério da Saúde considera como TDO a efetivação de no mínimo 24 tomadas, observadas por um profissional da equipe de saúde, na fase de ataque e 48 tomadas observadas na fase de manutenção, sendo que estas podem ser realizadas de forma compartilhada no domicílio do usuário e na unidade de saúde ${ }^{(4)}$.

Ações voltadas para prevenir o abandono de tratamento da TB são necessárias, uma vez que este aumenta o risco de resistência a antibióticos, transmissão de doenças, morbidade e mortalidade(25), devendo ser direcionadas principalmente às populações mais propensas ao abandono do tratamento como PVHA, moradores de rua, usuários de drogas, pessoas privadas de liberdade, entre outros ${ }^{(26)}$.

Os principais motivos que levam os pacientes coinfectados TB/HIV a abandonarem o tratamento da TB tem relação com o sentir-se curado após melhora dos sintomas, a não tolerância dos efeitos adversos dos medicamentos, a baixa condição socioeconômica e a impossibilidade de se ausentar no trabalho, limitando o comparecimento do mesmo ao serviço de saúde para consulta médica e para buscar os medicamentos, o uso de álcool e drogas ilícitas, que limitam a percepção do indivíduo em relação à importância da adesão ao tratamento. Deficiências relacionadas ao serviço de saúde como inadequada estrutura física, debilidades no quadro de recursos humanos e limitado acesso às ações e serviços de saúde também se mostraram relevantes para não adesão(27).

A classificação proposta para cobertura do TDO nos municípios paulistas foi essencial para explicar a possível relação deste com a ocorrência de territórios de risco. Neste sentido, municípios que possuem cobertura abaixo de $30 \%$ do TDO possivelmente são aqueles com menor incidência de casos de coinfecção, fazendo com que a política do TDO não seja implantada de forma efetiva pelos serviços de saúde, unicamente pelo fator de priorização a regiões com maior incidência dos casos.

Entretanto, municípios com cobertura entre 31 e $79 \%$ foram aqueles com maior 
probabilidade de formarem territórios de risco para o abandono, podendo significar que, apesar de serem locais mais endêmicos para a doença, não existe uma abrangência adequada desta cobertura para todos os pacientes, resultando em uma maior taxa do desfecho. Desta forma, comparativamente, municípios que possuem cobertura acima de $80 \%$ de TDO reduzem sua chance de serem territórios para o abandono do tratamento, representando maior capacidade de acesso dos pacientes com coinfecção ao TDO.

Para o óbito, as análises não identificaram relação com a cobertura do TDO. Importante enfatizar que muitos são os fatores que podem concorrer para o óbito de indivíduos com coinfecção TB/HIV, como potencial dos medicamentos, implementação de programas e os fatores clínicos que podem ser específicos para cada caso, o que pode explicar tanto a não associação com a cobertura do TDO como também a heterogeneidade na distribuição espacial encontrada nas análises espaciais. No entanto, as ações de saúde para a redução da mortalidade dos casos de coinfecção TB/HIV devem ser voltadas a essa população específica devido ao fato de essas pessoas terem maiores chances de evoluírem para o óbito, quando comparadas com PVHA sem TB ${ }^{(3)}$.

Apesar dos índices de mortalidade dos casos de coinfecção TB/HIV se apresentarem elevados, no Sistema Único de Saúde (SUS) há estratégias que favorecem a melhoria desse indicador, as quais vão além do oferecimento do TDO. Tais estratégias incluem a dispensação gratuita de tuberculostáticos e medicamentos antirretrovirais, atualização contínua de protocolos clínicos e diretrizes terapêuticas e prevenção da transmissão do HIV, por meio da oferta indiscriminada de preservativos e lubrificantes íntimos ${ }^{(28,29)}$.

Para o alcance das metas propostas pela estratégia End TB, há uma necessidade de melhorar o acesso ao diagnóstico da TB, melhorar o manejo da TB-Multi Droga Resistente, bem como as condições de coinfecção TB/HIV $(6,30)$. Para tanto, é necessário o desenvolvimento de pesquisas operacionais que têm papel relevante neste processo, pois apontam os desafios para a implementação das políticas públicas e fornecem evidências do ponto de vista dos pacientes e dos sistemas de saúde ${ }^{(30)}$.

Um dos principais desafios do controle da coinfecção TB/HIV se dá através da articulação dos programas de ambas as doenças ${ }^{(2)}$, pois o diagnóstico e tratamento desses pacientes ora se faz no Programa de Controle da TB ora no Programa de DST/AIDS. Sendo assim, o sucesso desses tratamentos pode estar ligado à gestão dos programas e suas articulações nas ações de TB e HIV.

O estudo apresenta limitações no que tange ao uso de dados secundários, que podem apresentar inconsistências e dados faltantes, apresentando assim viés de informação.

\section{CONCLUSÃO}

A RMSP e a Baixada Santista apresentam maior risco para a coinfecção, bem como para o abandono do tratamento da TB e óbitos entre esta população. O artigo avança na utilização de métodos estatísticos para analisar se esses fatores estavam associados à cobertura de TDO e identificou que a menor cobertura do TDO estava associada à formação de territórios de risco para a incidência da coinfecção TB/HIV e abandono do tratamento. Os achados apresentados podem auxiliar gestores na aplicação de políticas públicas voltadas a essas regiões, a fim de buscarem a efetivação do TDO e atingirem as populações que estão mais vulneráveis à coinfecção. 
1. World Health Organization (WHO). Global tuberculosis report 2017. Geneva: WHO; 2017 [acesso em 25 mar 2019]. Disponível em: https://www.aidsdatahub.org/sites/default/files/publication/WHO Global Tuberculosis Report 2017.pdf.

2. Ministério da Saúde (BR). Secretaria de Vigilância em Saúde. Departamento de Vigilância das Doenças Transmissíveis. Brasil livre da tuberculose: plano nacional pelo fim da tuberculose como problema de saúde pública [Internet]. Brasília: Ministério da Saúde; 2017 [acesso em 26 mar 2019]. Disponível em: http://bvsms.saude.gov.br/bvs/publicacoes/brasil livre tuberculose plano nacional.pdf.

3. World Health Organization, UNAIDS. Global report: UNAIDS report on the global AIDS epidemic 2013 [Internet]. Unaids. 2013. 198 p. [acesso em 26 mar 2019]. Disponível em: https://www.unaids.org/sites/ default/files/media asset/UNAIDS Global Report 2013 en 1.pdf.

4. Ministério da Saúde (BR). Secretaria de Vigilância em Saúde. Departamento de Vigilância das Doenças Transmissíveis. Manual de Recomendações para o Controle da Tuberculose no Brasil. Brasília (DF): Ministério da Saúde; 2018 [acesso em 16 fev 2019]. Disponível em: http://bvsms.saude.gov.br/bvs/ publicacoes/manual recomendacoes controle tuberculose brasil 2 ed.pdf.

5. Ministério da Saúde (BR). Secretaria de Vigilância em Saúde. Bol. Epidemiol. [Internet]. 2016 [acesso em 07 abr 2019]; 47(13). Disponível em: http://portalsaude.saude.gov.br/images/pdf/2016/marco/24/2016009-Tuberculose-001.pdf.

6. Unis G, Ribeiro AW, Esteves LS, Spies FS, Picon PD, Costa ERD, et al. Tuberculosis recurrence in a high incidence setting for HIV and tuberculosis in Brazil. BMC Infect Dis [Internet]. 2014 [acesso em 16 fev 2019]; 14. Disponível em: https://doi.org/10.1186/s12879-014-0548-6.

7. Matteelli A, Rendon A, Tiberi S, Al-Abri S, Voniatis C, Carvalho ACC, et al. Tuberculosis elimination: Where are we now? Eur Respir Rev [Internet]. 2018 [acesso em 23 jan 2019]; 27(148):1-15. Disponível em: http://dx.doi.org/10.1183/16000617.0035-2018.

8. Rothman K, Greenland S, Lash T. Epidemiologia Moderna. 3 ed. Artmed. 2011.

9. Ministério da Saúde (BR). Departamento de Informática do SUS (BR). Informações de saúde: epidemiológicas e morbidade - 2015 [Internet]. Brasília (DF): Ministério da Saúde; 2016 [acesso em 07 mar 2019]. Disponível em: http://www2.datasus.gov.br/DATASUS/index.php?area=0203.

10. Gordis L. Epidemiology. 5 ed. Systematic biology; 2013. 416 p.

11. Jenks G. The data model concept in statistical mapping. Int Yearb Cartogr.; 1967.

12. Anselin L. Local Indicators of Spatial Association-LISA. Geogr Anal.; 1995.

13. Tobler W. A Computer Movie Simulating Urban Growth in the Detroit Region. Econ Geogr. [Internet]. 1970 [acesso em 26 mar 2019]. Disponível em: http://doi.org/10.2307/143141.

14. Arslan H. Spatial and temporal mapping of groundwater salinity using ordinary kriging and indicator kriging: The case of Bafra Plain, Turkey. Agric Water Manag. [Internet]. 2012 [acesso em 23 jan 2019]; 113:57-63. Disponível em: https://doi.org/10.1016/j.agwat.2012.06.015.

15. Isaaks EH, Srivastava R. Applied Geostatistics. Press OU. New York: Oxford University Press; 1989. 561 p.

16. Sibson R. A brief description of natural neighbor interpolation. In: Interpreting Multivariate Data; 1981.

17. Kulldorff M, Nagarwalla N. Spatial disease clusters: Detection and inference. Stat Med. [Internet]. 1995 [acesso em 26 mar 2019]; 14(8):799-810. Disponível em: https://doi.org/10.1002/sim.4780140809. 
18. Han J, Zhu L, Kulldorff M, Hostovich S, Stinchcomb DG, Tatalovich Z, et al. Using Gini coefficient to determining optimal cluster reporting sizes for spatial scan statistics. Int J Health Geogr [Internet]. 2016 [acesso em 16 fev 2019]; 15(1). Disponível em: https://doi.org/10.1186/s12942-016-0056-6.

19. Besag J, York J, Mollié A. Bayesian image restoration, with two applications in spatial statistics. Ann I Stat Math. [Internet]. 1991 [acesso em 16 fev 2019]; 43(1):1-20. Disponível em: https://doi.org/10.1007/ BF00116466.

20. Rue H, Martino S, Lindgren F, Simpson D, Riebler A, Krainski E. INLA: Functions Which Allow to Perform Full Bayesian Analysis of Latent Gaussian Models Using Integrated Nested Laplace Approximaxion. R package version 0.0-1389624686. 2014.

21. Oliveira RNF. As Políticas Públicas de Educação e Saúde como integrantes dos índices de vulnerabilidade social e condicionantes da prosperidade social [dissertação]. NOVA Information Management School: Universidade Nova Lisboa; 2018. Disponível em: http://hdl.handle. net/10362/31398.

22. Nunes C, Duarte R, Veiga AM, Taylor B. Who are the patients that default tuberculosis treatment? space matters! Epidemiol Infect [Internet]. 2017 [acesso em 07 mar 2019]; 145(6):1130-4. Disponível em: https://doi.org/10.1017/S0950268816003307.

23. Tian JH, Lu ZX, Bachmann MO, Song F. Effectiveness of directly observed treatment of tuberculosis: A systematic review of controlled studies. Int J Tuberc Lung Dis [Internet]. 2014 [acesso em 07 mar 2019]; 18(9):1092-8. Disponível em: https://doi.org/10.5588/ijtld.13.0867.

24. Ibanês AS, Carneiro Junior N. Panorama internacional e nacional da estratégia do tratamento diretamente supervisionado (DOTS) nas políticas de controle da tuberculose. ABCS Health Sci [Internet]. 2013 [acesso em 17 fev 2019]; 38(1):25-32. Disponível em: https://doi.org/10.7322/abcshs.v38i1.5.

25. Grzybowski S, Enarson DA. The fate of cases of pulmonary tuberculosis under various treatment programmes. Bull Int Union Tuberc. [Internet]. 1978 [acesso em 23 jan 2019]; 53(2):70-5. Disponível em: https://www.cabdirect.org/cabdirect/abstract/19782902484.

26. Ministério da Saúde (BR). Secretaria de Vigilância em Saúde. Departamento de Vigilância das Doenças Transmissíveis. Saúde Brasil 2013 : uma análise da situação de saúde e das doenças transmissíveis relacionadas à pobreza. [Internet]. Brasília: Ministério da Saúde; 2014 [acesso em 26 abr 2019]. Disponível em: http://bvsms.saude.gov.br/bvs/publicacoes/saude brasil 2013 analise situacao saude.pdf.

27. Rodrigues ILA, Monteiro LL, Pacheco RHB, Silva SÉD da. Abandonment of tuberculosis treatment among patients co-infected with TB/HIV. Rev Esc Enferm USP [Internet]. 2010 [acesso em 12 abr 2019]; 44(2):383-7. Disponível em: http://dx.doi.org/10.1590/S0080-62342010000200020.

28. Ministério da Saúde (BR). Secretaria de Vigilância em Saúde. Departamento de DST/Aids. Boletim Epidemiológico: Aids e DST. 2014; 4 (3):1-84.

29. Trajman A, Pinto M, Souza R, Freitas M, Torrens A, Lima J, et al. Market assessment of tuberculosis diagnostics in Brazil in 2012. PLoS One [Internet]. 2014 [acesso em 14 mar 2019]; 9(8):e104105. Disponível em: https://doi.org/10.1371/journal.pone.0104105.

30. Lienhardt C, Lönnroth K, Menzies D, Balasegaram H, Chakaya J, Cobelens F, et al. Translational research for tuberculosis elimination: priorities, challenges, and actions. PLOS Med [Internet]. 2016 [acesso em 23 mar 2019]; 13(3):e1001965. Disponível em: https://doi.org/10.1371/journal.pmed.1001965

Recebido: $13 / 05 / 2019$

Finalizado: 25/09/2019

Autor Correspondente:

Laura Terenciani Campoy 
Universidade de São Paulo

Av. dos Bandeirantes, 3900 - 14040-902 - Ribeirão Preto, SP, Brasil

E-mail: lauratcampoy@hotmail.com

Contribuição dos autores:

Contribuições substanciais para a concepção ou desenho do estudo; ou a aquisição, análise ou interpretação de dados do estudo - LTC, LHA, MAMA

Elaboração e revisão crítica do conteúdo intelectual do estudo - LTC, LHA, ACVR, RLPA

Aprovação da versão final do estudo a ser publicado - LTC, LHA, ACVR, RLPA, MAMA, JDA, RAA

Responsável por todos os aspectos do estudo, assegurando as questões de precisão ou integridade de qualquer parte do estudo - LTC, LHA, ACVR, RLPA, MAMA, JDA, RAA 